\title{
Malestar psicológico en mujeres con embarazo de alto riesgo
}

\section{Psychological distress in high-risk pregnant women}

\author{
María Eugenia Gómez López \\ Instituto Nacional de Perinatología "Isidro Espinosa de los Reyes", México \\ Shoshana Berenzon Gorn \\ María Asunción Lara \\ Instituto Nacional de Psiquiatría "Ramón de la Fuente Muñíz", México \\ María Emily Ito Sugiyama \\ Universidad Nacional Autónoma de México \\ (Rec.: febrero de 2016 - Acept.: abril de 2016)
}

\begin{abstract}
Resumen
La experiencia emocional de la mujer con embarazo de alto riesgo ante la maternidad es un tema poco estudiado, a pesar de la importancia que tiene preservar su salud emocional durante este proceso. Esta investigación analizó la experiencia de malestar psicológico en mujeres con embarazo de alto riesgo, según el riesgo médico diagnosticado, su vivencia de la maternidad y el papel que juegan la pareja, familia y redes de apoyo durante la gestación. Se realizó un estudio instrumental de casos múltiples. Se hicieron entrevistas semiestructuradas a 12 mujeres adultas con diagnóstico médico de embarazo de alto riesgo y se formaron tres grupos. Se realizó un análisis de significados de Kvale de los datos obtenidos. Los resultados mostraron que la vivencia de la maternidad y la manifestación de malestar psicológico en estas mujeres era diferente, dependiendo del riesgo médico diagnosticado. Además, podía ser de menor o mayor intensidad debido a la falta de planeación del embarazo, las experiencias obstétricas previas, no tener hijos vivos y contar con el apoyo de la pareja y la familia. Las mujeres con embarazo de alto riesgo vivían la maternidad como una experiencia dolorosa, con mucha angustia y miedo de perder el nuevo embarazo por complicaciones.
\end{abstract}

Palabras clave: embarazo de alto riesgo, maternidad, malestar psicológico.

\begin{abstract}
The emotional experience of maternity in high-risk pregnant women is a subject that has been seldom studied, despite the importance of preserving the emotional health of women during this stage. This research analyzed the experience of psychological distress in women with high-risk pregnancy according to the medical risk diagnosed, their experience of maternity and the role of the partner, family and support networks during pregnancy. An instrumental multiple cases study was performed. By semi-structured interviews, we interviewed 12 adults' women with medical diagnosis of high-risk pregnancy and three groups were formed. A Kvale analysis of meanings was performed on data. The results showed that the experience of motherhood and the manifestation of psychological distress in these women were different depending on the diagnosed medical risk; it may be of lower or higher intensity due to the lack of family planning, previous obstetric experiences, the lack of living children and also the support of the partner and the family. High-risk pregnant women lived mateity as a painful experience, with great anxiety and fear of losing the new pregnancy for medical complications.
\end{abstract}

Keywords: high-risk pregnancy, maternity, psychological distress.

\footnotetext{
* Correspondencia a: María Eugenia Gómez López. Montes Urales 800, Lomas Virreyes, Del. Miguel Hidalgo, 11000 Ciudad de México, México. E-mail: eugeniagomez2712@yahoo.com.mx
} 


\section{Introducción}

La experiencia emocional de la mujer frente a un embarazo de alto riesgo es un tema poco estudiado por la literatura mundial. En su abordaje ha prevalecido el enfoque biomédico, el cual explica la enfermedad en función de los síntomas físicos, dejando de lado sus dimensiones sociales, psicológicas y conductuales.

Desde el enfoque biomédico, el interés por investigar este tema se ha orientado principalmente hacia las mujeres hospitalizadas, cuyos principales hallazgos a nivel emocional han sido que las complicaciones obstétricas presentadas por ellas pueden llegar a provocarles ansiedad por la salud y bienestar del feto, dificultad para manejar el confinamiento en el hospital, una sensación de estar "prisionera", así como sentimientos de vulnerabilidad y soledad. Junto a ello, pueden presentar estrés y ansiedad por no ser capaces de realizar sus actividades normales, además de manifestaciones de tristeza, incertidumbre y culpa por estar separadas de su hogar y de su familia (Hediye \& Korkmaz, 2005; Peixoto, Carvalho \& Lefèvre, 2006).

Otros hallazgos de investigación señalan que también algunas complicaciones perinatales como la preeclampsia, el retardo en el crecimiento uterino, el bajo peso del infante al nacer y el parto prematuro, se asocian con factores psicosociales como el estrés, los conflictos de pareja, la autoestima de la madre, la falta de apoyo social y el bajo nivel socioeconómico en la manifestación de depresión o ansiedad durante el embarazo (Dennis, 2005; Lara, Navarro \& Navarrete, 2010).

Sin embargo, desde el enfoque biomédico, la visión que prevalece de la mujer embarazada, en cuanto a su salud mental, es que ella se encuentra más vulnerable, está en riesgo, en desventaja y por tanto, es más susceptible de enfermarse mentalmente. Además, el embarazo se ve como un "evento negativo", "una etapa estresante, complicada, de crisis", "un detonador", que puede llegar a enfermar mentalmente a las mujeres que lo viven.

Esto ha provocado que las manifestaciones emocionales que algunas mujeres pueden presentar durante la gestación lleguen a sobrediagnosticarse como ansiedad o depresión; aun cuando éstas no reúnen las características para diagnosticarse como un trastorno y son más bien manifestaciones emocionales de tipo depresivo y ansioso temporales y esperadas, en respuesta al ajuste que las personas hacen frente a las crisis de la vida. A dichas manifestaciones se les llama malestar psicológico (Austin \& Priest, 2005; Fisher, Feekery \& Rowe-Murray, 2002; Glazier, Elgar, Goel, \& Holzapfel, 2004).

Estudios realizados en México sobre el malestar psicológico en poblaciones obstétricas de alto riesgo seña- lan una prevalencia de $62 \%$. Los principales hallazgos sobre este tema plantean que este evento reproductivo moviliza distintas reacciones emocionales en la mujer por la importancia que tiene en su vida, y que alguna complicación durante la gestación puede generar sintomatología ansiosa o depresiva (Gómez, 2007; Gómez \& Aldana, 2007; Gómez, Morales, Aldana \& Gaviño, 2008).

Sin embargo, frente a esta perspectiva biomédica de la salud mental de la mujer embarazada, existe un enfoque alternativo, más crítico e interpretativo, contra la medicalización de la salud mental en general, y que propone la necesidad de generar otros modelos que sean complementarios y que se enfoquen en el estudio de la salud mental de la mujer, en particular, que alejen este concepto de lo psicopatológico para acercarlo al escenario de la vida cotidiana (Burin, 1990; Castro, 2000; Velasco, 2006).

Este enfoque crítico permite profundizar en la experiencia emocional de la mujer con embarazo de alto riesgo para conocer la subjetividad de su vivencia, su experiencia, sus sentimientos y percepciones frente a las complicaciones durante la gestación.

Desde este enfoque crítico e interpretativo, se han realizado algunas investigaciones para comprender la condición emocional que viven las mujeres hospitalizadas por complicaciones en sus embarazos, la cual se caracteriza principalmente por sentimientos de ambivalencia, tristeza, preocupación, inseguridad y angustia, además de que pueden llegar a manifestar mucho temor por la posibilidad de perder a su hijo (Pinho, Pamplona \& Garcia de Lima, 2010; Lafaurie et al., 2011; Laza, Quintero, Jiménez \& Preciado, 2013).

Al respecto, cabe destacar las aportaciones de Burin (1990) alrededor de la construcción de la subjetividad femenina y de su salud mental en la cultura actual, creando con la maternidad, la base de su subjetividad. Esta autora afirma que dicha situación provoca conflictos a las mujeres, expresados a través del malestar, es decir, manifestaciones emocionales transitorias de diversa índole que se presentan en respuesta a distintas situaciones de la vida, del contexto social. La experiencia y vivencia de éstas, dependen de la subjetividad de cada persona, y aunque tienden a "naturalizarse" y a verse como algo "normal", generan importantes costos de salud y bienestar en la población.

Al respecto, Burin (1990) plantea además que una de las formas en que las mujeres manifiestan los trastornos de la vida cotidiana y expresan su malestar es a través de sus síntomas.

Esta postura es importante porque además de la visión biomédica del embarazo, incorpora una mirada en la que las mujeres expresan sus conflictos subjetivos en torno a la maternidad y a su identidad, a través de un 
Malestar psicológico en mujeres con embarazo de ALto RiEsGo

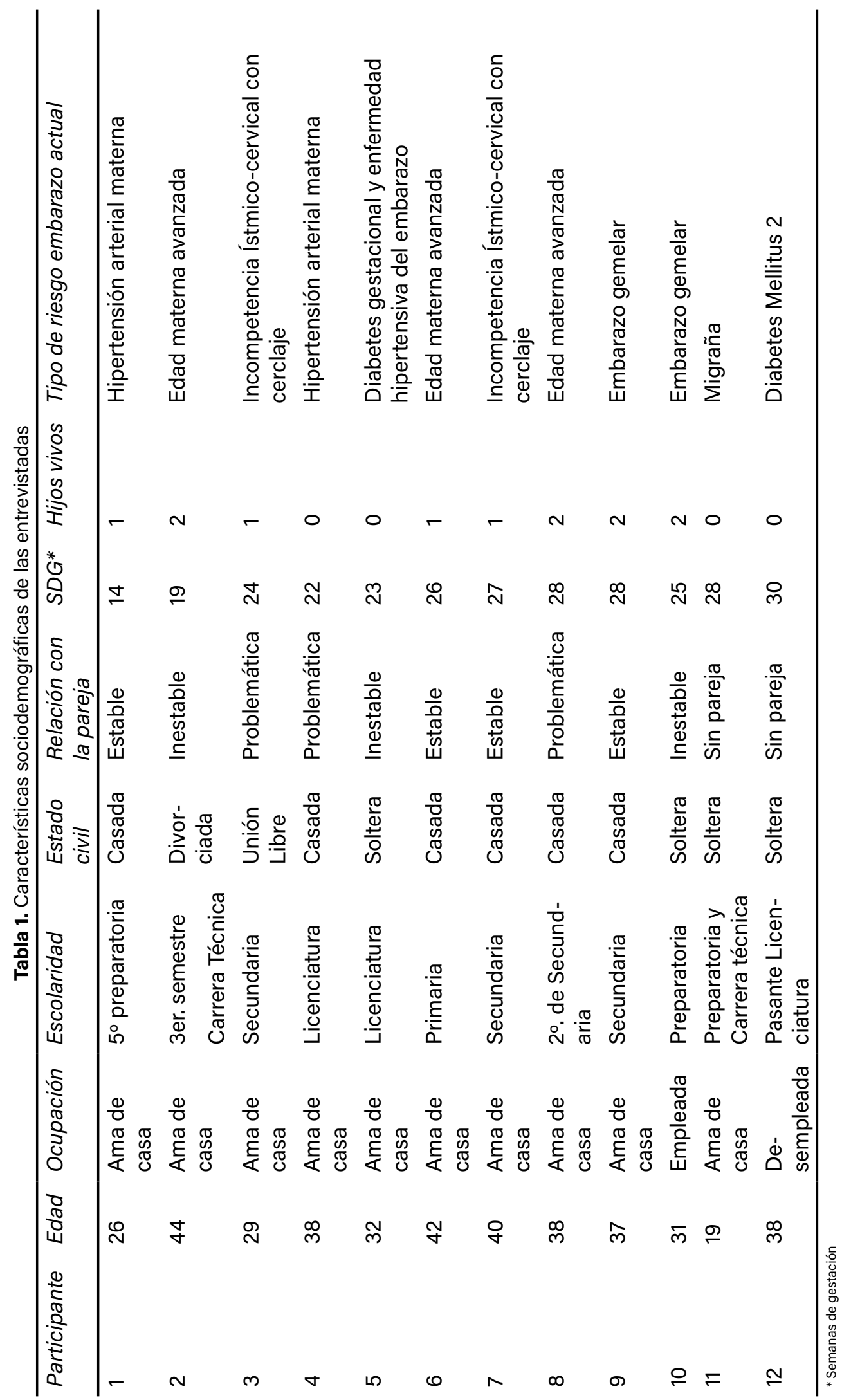


malestar de la vida cotidiana.

Con base en estos planteamientos de Burin (1990) y en los supuestos teóricos del interaccionismo interpretativo propuesto por Denzin (2001), se realizó una investigación cualitativa interpretativa. Esta última perspectiva se enfoca en aquellas experiencias de vida que alteran y moldean radicalmente los significados que las personas dan a ellos mismos y a sus experiencias. De esta manera, el objetivo de este estudio fue comprender la experiencia de malestar psicológico en las mujeres con embarazo de alto riesgo, a partir del tipo de riesgo médico diagnosticado (enfermedad previa al embarazo, complicaciones del embarazo y otros factores de riesgo), su vivencia de la maternidad y el papel que juegan la pareja, la familia y las redes de apoyo durante este evento reproductivo.

El presente trabajo forma parte de un estudio más amplio del que se retomaron algunos temas que pueden considerarse centrales para la comprensión del fenómeno abordado.

\section{Metodología}

\section{Población}

En este estudio participaron 12 mujeres mayores de 18 años de edad, pacientes de un hospital de tercer nivel especializado en la atención obstétrica de la Ciudad de México, quienes fueron invitadas a participar voluntariamente, y se les eligió con base en el tipo de riesgo gestacional diagnosticado médicamente.

Se utilizó un diseño de estudio instrumental de casos múltiples (Cresswell, 1998; Martínez, 2006; Stake, 2005).

Se formaron tres grupos: 1) mujeres con enfermedades previas al embarazo (con padecimientos crónicos que las predisponían a tener problemas durante el embarazo); 2) mujeres con complicaciones en el embarazo actual (aquellas que desarrollaron una complicación imprevista durante un embarazo que previamente era normal); y 3) mujeres con otros factores de riesgo asociados al embarazo (factores potenciales de riesgo de alguna complicación durante el embarazo por tener edad materna avanzada, embarazo múltiple, entre otros). En la tabla 1 (ver página anterior) se presentan las principales características sociodemográficas de las participantes.

\section{Procedimiento}

Se invitó a participar en el estudio a aquellas mujeres embarazadas con diagnóstico médico de alto riesgo, a quienes se les solicitó el consentimiento informado de manera verbal y se les pidió autorización para audiograbarlas.

Se realizaron entrevistas semiestructuradas, dado que se consideran una técnica para reconstruir las teorías subjetivas. A través de ellas, se intenta comprender el mundo desde el punto de vista de los sujetos, para dar a conocer el significado de las experiencias de las personas, descubrir su mundo vivido antes de las explicaciones científicas (Flick, 2004).

Con este fin, se elaboró una guía de entrevista ${ }^{1}$ con la secuencia de temas (Kvale, 1996), a partir de los objetivos. En este estudio sólo se analizaron los siguientes temas clave: 1) Malestar, 2) Experiencia de la maternidad y 3) Relaciones interpersonales.

Las entrevistas se realizaron en un lugar cómodo y aislado del ruido que permitiera hacerlas en las mejores condiciones y tuvieron una duración aproximada de una hora.

\section{Análisis de la información}

En un primer momento, se transcribieron las entrevistas $y$, posteriormente fueron analizadas con base en la técnica de categorización de significados ${ }^{2}$ de Kvale (1996). La información obtenida a partir de los relatos registrados mediante la entrevista, se clasificó primero en las tres categorías de interés, y después se separó por subcategorías para reunir los datos de las 12 entrevistas (véase la tabla 2). En la tabla 3 se muestra la definición de las categorías analizadas así como las subcategorías obtenidas.

Tabla 2. Entrevistas realizadas

\begin{tabular}{|c|c|}
\hline Tipo de riesgo & Participantes \\
\hline \multirow{3}{*}{$\begin{array}{l}\text { Enfermedad previa } \\
\text { al embarazo actual }\end{array}$} & $\begin{array}{l}2 \text { mujeres con hipertensión } \\
\text { arterial, antecedente de pre- } \\
\text { eclampsia }\end{array}$ \\
\hline & 1 mujer con migraña \\
\hline & 1 mujer con diabetes mellitus \\
\hline \multirow{2}{*}{$\begin{array}{l}\text { Complicaciones del } \\
\text { embarazo }\end{array}$} & $\begin{array}{l}2 \text { mujeres con incompetencia } \\
\text { ístmico-cervical }\end{array}$ \\
\hline & $\begin{array}{l}1 \text { mujer con diabetes gesta- } \\
\text { cional }\end{array}$ \\
\hline \multirow{2}{*}{$\begin{array}{l}\text { Otros factores de } \\
\text { riesgo }\end{array}$} & $\begin{array}{l}3 \text { mujeres con edad materna } \\
\text { avanzada }\end{array}$ \\
\hline & $\begin{array}{l}2 \text { mujeres con embarazo ge- } \\
\text { melar }\end{array}$ \\
\hline
\end{tabular}

Fuente: Elaboración propia

\footnotetext{
${ }^{1}$ Una guía de entrevista menciona varias áreas temáticas. Cada una de ellas se introduce por una pregunta abierta y se finaliza por una pregunta de confrontación para reconstruir el contenido de la teoría subjetiva (Flick, 2004).

2 Por medio de esta técnica, cada entrevista se codifica en una serie de categorías mutuamente excluyentes, lo que permite estructurar las narraciones en unidades de información que facilitan la comprensión del tema de interés, así como su ocurrencia a lo largo del discurso (Berenzon, Saavedra y Alanís, 2009).
} 
Tabla 3. Categorías y subcategorías obtenidas

\begin{tabular}{|c|c|c|c|}
\hline Categoría & Definición & Subcategorías & Definición \\
\hline \multirow{3}{*}{ Malestar } & \multirow{3}{*}{$\begin{array}{l}\text { Manifestaciones emocionales displa- } \\
\text { centeras transitorias que señalaron las } \\
\text { mujeres alrededor de dos momentos } \\
\text { críticos o epifanías: 1) la noticia del } \\
\text { embarazo y 2) el diagnóstico médico de } \\
\text { alto riesgo. Ambas situaciones les gen- } \\
\text { eraron malestares físicos, emocionales y } \\
\text { físicos-emocionales. }\end{array}$} & $\begin{array}{l}\text { 1. } 1^{\text {a }} \text { epifanía: noticia del } \\
\text { embarazo }\end{array}$ & $\begin{array}{l}\text { Manifestaciones emocio- } \\
\text { nales frente al diagnóstico } \\
\text { médico del embarazo } \\
\text { actual. }\end{array}$ \\
\hline & & $\begin{array}{l}\text { 2. } 2^{\text {a }} \text { epifanía: Vivencia del } \\
\text { diagnóstico de riesgo }\end{array}$ & $\begin{array}{l}\text { Manifestaciones emociona- } \\
\text { les asociadas al diagnóstico } \\
\text { médico de alto riesgo. }\end{array}$ \\
\hline & & $\begin{array}{l}\text { 3. Repercusiones } \\
\text { físicas, emocionales y } \\
\text { físicas-emocionales. }\end{array}$ & $\begin{array}{l}\text { Vivencia de los malestares } \\
\text { físicos, emocionales y físico- } \\
\text { emocionales experimen- } \\
\text { tados por las mujeres a } \\
\text { partir de los dos momentos } \\
\text { críticos. }\end{array}$ \\
\hline
\end{tabular}

Las vivencias de las mujeres en embarazos previos, su condición de salud Experiencia de la y su influencia en la percepción y las maternidad expectativas hacia la maternidad y en el significado que para ellas tenía el riesgo de su embarazo.

La relación de las mujeres entrevistadas con su pareja, su familia y sus redes de apoyo, al momento del embarazo y a partir del diagnóstico de riesgo.

\begin{tabular}{ll} 
1. Pareja & $\begin{array}{l}\text { Percepción de las mujeres } \\
\text { sobre la relación con su } \\
\text { pareja y el impacto emocio- } \\
\text { nal vivido por el embarazo } \\
\text { de alto riesgo y malas expe- } \\
\text { riencias obstétricas previas. }\end{array}$ \\
\hline 2. Familia & $\begin{array}{l}\text { Percepción del apoyo de } \\
\text { la familia en el embarazo } \\
\text { de alto riesgo y el impacto } \\
\text { emocional vivido por malas } \\
\text { experiencias obstétricas } \\
\text { previas. }\end{array}$ \\
\hline 3. Redes de apoyo & $\begin{array}{l}\text { Percepción de las reac- } \\
\text { ciones de las personas } \\
\text { cercanas (amigos y vecinos) } \\
\text { a partir del embarazo. }\end{array}$
\end{tabular}

Relaciones interpersonales a partir del embarazo.

\section{Consideraciones éticas}

Se obtuvo el consentimiento informado de forma verbal de cada una de las mujeres entrevistadas. La investigadora les explicó detalladamente el objetivo del estudio, en qué consistía su participación, la duración de la misma, así como su carácter voluntario, ya que podían aceptar y retirar su participación en el momento en que lo desearan. Se verificó que las participantes hubieran comprendido adecuadamente la información proporcionada y se les resolvieron sus dudas. Se les pidió autorización para audiograbar la entrevista. Se les explicaron los beneficios y los riesgos de su participación en el estudio, y se les mencionó que si durante la entrevista se detectaba alguna alteración de su estado de ánimo que requiriera de atención psicológica, ésta se le brindaría de manera gratuita dentro de la insti- tución. Se hizo hincapié en la confidencialidad tanto de la información como de su identidad, por lo que los nombres verdaderos de las participantes fueron cambiados por seudónimos. Esta investigación se consideró de riesgo mínimo, ya que solamente se entrevistaba a las participantes y lo que podría llegar a presentarse durante la sesión sería alguna alteración del estado de ánimo, producto de lo evocado durante la entrevista (Meo, 2010; National Institute of Health, 2008).

\section{Resultados}

A continuación se muestran los resultados obtenidos en cada categoría, en donde se compara la información obtenida en cada grupo de riesgo médico, buscando tanto sus coincidencias como sus diferencias. 


\section{Malestar}

Para comprender los resultados obtenidos en esta categoría, es importante explicar que éstos se organizaron alrededor de dos momentos de crisis o epifanías (Denzin, 2001) vividos por estas mujeres: el primero, al enterarse de su embarazo, noticia que las tomó por sorpresa (dado que no había sido planeado ni por ellas ni por su pareja); y el segundo, cuando el médico les diagnosticó un embarazo de alto riesgo.

Estos dos momentos generaron en las participantes la manifestación de diversas emociones que abarcaban desde la alegría hasta el temor, el miedo y la angustia de repetir experiencias previas de pérdida y complicaciones graves de su salud que llegaron incluso a poner en riesgo sus vidas y las de sus hijos. A partir de estas epifanías, estas mujeres experimentaron también diversos cambios físicos, emocionales y físico-emocionales, aspectos que se incluyen en una tercera subcategoría.

\subsection{1a Epifanía: Noticia del embarazo}

A decir de las participantes, se presentaron dos situaciones alrededor de su embarazo: 1) no lo planearon, o 2) tomaron la decisión de embarazarse sin consultar con su pareja. Sin embargo, todas manifestaron que su diagnóstico fue algo sorpresivo.

Para las mujeres con enfermedades crónicas previas, el hecho de que el embarazo fuera inesperado, no planeado o por decisión propia sin consultar a su pareja, fue algo sorpresivo e impactante, ya que para algunas esto se asociaba con experiencias obstétricas previas que fueron negativas.

Dichas vivencias, junto con la gravedad del riesgo médico del embarazo actual, les generaba una situación ambivalente entre la angustia de volver a vivir experiencias negativas, la posibilidad de perder a su bebé y la alegría del nuevo embarazo.

No lo esperaba, lo tomé con angustia. Antes del embarazo habíamos platicado (mi pareja y yo) que ya no, él me dijo: si se te complica, ya sabemos lo que es ser padres, mejor ya no. (Entrevistada 1)

Otras lo vivieron como una devastación emocional, con miedo por su salud, así como enojo y rechazo por su condición.

Algunas mujeres del grupo con complicaciones del embarazo decidieron por sí mismas embarazarse sin consultar a la pareja y otras no lo planearon. Aquellas mujeres que se embarazaron por decisión propia manifestaron que, a través del hijo, podrían salvar su relación de pareja o cumplir el sueño de toda su vida:

Este... sí, en cierta parte sí yo pensé que con el embarazo él (su pareja) iba a cambiar un poco. (Entrevistada 3)

Las principales emociones manifestadas por quienes no planearon el embarazo, fueron de alegría o de ambivalencia entre "alegría y miedo a la pérdida", y "angustia y miedo a la pérdida".

En cuanto a las mujeres con otros factores de riesgo, éstas vivieron con sorpresa e impacto el embarazo inesperado:

me cayó de golpe, porque ya no estaba en mi proyecto volver a tener bebés. (Entrevistada 10)

\subsection{2a Epifanía: Vivencia del diagnóstico de riesgo}

Para algunas mujeres con enfermedades crónicas previas, el riesgo médico existente fue originado por las experiencias obstétricas negativas previas, las que a su vez les ocasionaron, entre otras, hipertensión arterial. Esto las ponía en riesgo de desarrollar preeclampsia en su embarazo actual, con la posibilidad de perder al producto o de tener que interrumpirlo porque ponía en riesgo su vida:

No, de allí (embarazo anterior) quedé hipertensa. Pues yo no me sentía mal, a míme regulan la presión; es cuando yo me siento mal cuando se me sube. (Entrevistada 1)

Por esto, frente al diagnóstico del embarazo, estas mujeres respondieron a nivel emocional, con angustia, tristeza, ambivalencia -entre alegría y angustia-, impacto, preocupación, devastación emocional, incertidumbre por su estado de salud y enojo, así como nerviosismo, temor a las complicaciones, preocupación por tener la atención médica, miedo a perder el embarazo, estar pendiente de los movimientos fetales, inquietud al comprender la magnitud del riesgo, sentirse disminuida por los problemas de salud, culpabilidad por no haberse embarazado antes:

se me hizo muy complicado y fue una situación que me devastó mucho emocionalmente, porque pensar en ser madre en ese escenario, fue así como que dije ¡no!. (Entrevistada 12)

Por su parte, las principales complicaciones médicas presentadas por las mujeres con complicaciones durante el embarazo fueron la incompetencia ístmico-cervical con cerclaje (lo que les había provocado diversas pérdidas gestacionales previas) y la diabetes gestacional. Cuando se les diagnosticó este tipo de riesgo, las mujeres reaccionaron con miedo a perder el producto, angustia y miedo por la salud, temor de repetir experiencias negativas previas, enojo, dificultad para aceptar el embarazo, culpa por la enfermedad actual, reproche, castigo, ocultar el estado real de salud a la familia, presión, preocupación y necesidad de cuidarse más:

Me reproché mucho, me dio mucho coraje conmigo, de hecho sigo enojada conmigo... (Entrevistada 5)

A las mujeres del grupo otros factores de riesgo en el embarazo, les diagnosticaron edad materna avanzada y embarazo gemelar, los cuales sólo representaban 
riesgos médicos potenciales (es decir, no padecían ninguna enfermedad o complicación que pusiera en riesgo su salud ni la de su bebé) y ninguna de ellas presentó problemas previos de salud. Por esto, su reacción emocional frente al diagnóstico de riesgo fue menos intensa que las de los otros grupos y se caracterizó por sorpresa, gusto, impacto, preocupación, dificultad de aceptación, enojo y aceptación:

...pero sí sentí: "¿y ahora qué voy a hacer?, jotra vez a volver a empezar! Cuando me van diciendo que son dos, iyyyy!, pues síme cayó así como de peso. (Entrevistada 10)

Se pudo observar que dependiendo de la gravedad del riesgo médico diagnosticado, fue la intensidad de la manifestación emocional de las participantes. En este sentido, la angustia y el miedo que sentían las mujeres del grupo con enfermedades crónicas previas estaban centrados en su salud y su vida, y las de su bebé. Aunque también, algunas presentaban una ambivalencia entre la alegría por el embarazo y el temor por volver a tener complicaciones. Por su parte, para las mujeres del grupo de complicaciones del embarazo, la angustia y el miedo se enfocaban en el temor a perder este bebé y a repetir esa experiencia de pérdida gestacional, aunque algunas sentían emociones ambivalentes entre la alegría y el temor a tener otra pérdida. Por su parte, las mujeres del grupo de otros factores de riesgo no vivían su embarazo como de alto riesgo, por lo que sus manifestaciones emocionales tenían que ver más con situaciones de su entorno (economía, empleo, pareja). Se observó que pese a eso, ellas también sintieron alegría por su nuevo embarazo.

\subsection{Repercusiones en las mujeres: físicas, emociona- les y físicas-emocionales}

Las principales repercusiones del embarazo inesperado y del diagnóstico de alto riesgo en las participantes durante su embarazo fueron: físicas, emocionales y físicas-emocionales.

En cuanto a las repercusiones físicas, las participantes de los tres grupos de riesgo proporcionaron respuestas similares respecto de los cambios y malestares físicos que habían tenido y que coincidían con los más comunes del embarazo reportados por la literatura médica, como son: náuseas, piernas hinchadas, dificultades para dormir, cansancio, dolor en senos, cefalea, mareos, entre otros. Quienes padecían diabetes reportaron, además, síntomas como caída de pelo y resequedad en la piel y boca, propios de esta condición. Sin embargo, el impacto a nivel emocional y físico-emocional fue diferente en los tres grupos.

Las mujeres del grupo de enfermedades crónicas previas refirieron como repercusiones emocionales haber sentido angustia, temor, miedo a las complicaciones, aprensión, preocupación por el futuro, hipersensibilidad, preocupación por la salud:
Pues el temor, que yo ya sé a lo que vengo, ya sé lo que puede suceder. (Entrevistada 1)

Como malestar físico-emocional, una participante mencionó que cada vez que acudía a consulta médica, se le subía la presión arterial por la angustia de sus complicaciones.

Por su parte, las mujeres con complicaciones del embarazo manifestaron como repercusiones emocionales haber presentado cambios de humor, enojo, irritabilidad, miedo a la soledad, melancolía y tristeza. En este grupo no se identificó ninguna repercusión físico-emocional.

Para las mujeres del grupo otros factores de riesgo, las repercusiones emocionales más relevantes fueron ánimo bajo, desgano, aunque también mostraron mayor conciencia de autocuidado. A nivel físico-emocional, una de las participantes mencionó haber tenido una dermatitis nerviosa ocasionada por el diagnóstico de embarazo gemelar:

Cuando me van diciendo que son dos, hasta de la impresión me dio dermatitis severa, toda me enronché. (Entrevistada 10)

En esta subcategoría, puede apreciarse nuevamente que con respecto a los malestares emocionales alrededor del embarazo de alto riesgo, los tres grupos de mujeres refieren haber sentido emociones que difieren en la intensidad, que van desde sentir ánimo bajo y desgano (otros factores de riesgo), hasta la angustia, el temor y el miedo a las complicaciones, de acuerdo con el riesgo médico presentado y lo que ello representaba para su salud (enfermedades crónicas previas y complicaciones del embarazo).

\section{Experiencia de la maternidad}

Para las mujeres con enfermedades crónicas previas y con complicaciones del embarazo, su percepción o vivencia acerca de la maternidad dependía de sus experiencias previas, la cual se dividió en: a) quienes tenían hijos vivos, b) quienes no los tenían, y c) quienes estaban viviendo su primer embarazo. No obstante, la diferencia entre las experiencias estaba dada por el riesgo que implicaba un embarazo para la salud de las mujeres del primer grupo y el temor a una nueva pérdida para las mujeres del segundo grupo:

Mmmh... pues... nada comparado con lo que me ha sucedido...", "una experiencia dolorosa, no tener nada, quedarse vacía. (Entrevistada 4)

En este grupo además, para quienes tenían hijos vivos, la vivencia de la maternidad se caracterizaba por experiencias negativas que provocaban enojo, exigencia y desesperación por los hijos; experiencias dolorosas por las pérdidas anteriores, llegando a pensar que la maternidad era algo negado y experiencias positivas de ser madre: 
siento gusto por saber que voy a tener un hijo... pero ante todo está el miedo, el miedo... (Entrevistada 7)

En cuanto a quienes vivían su primer embarazo, su percepción acerca de la maternidad era negativa por tener que vivirla sola y con toda la responsabilidad, pero al mismo tiempo, positiva ya que estaba cumpliendo un sueño:

¿Cuál es mi sueño en la vida? Ser mamá, o sea, no ser esposa... ser mamá (enfatiza). (Entrevistada 5)

Por otra parte, para el grupo de otros factores de riesgo, la percepción o vivencia de la maternidad giraba alrededor de las experiencias que tenían con sus hijos vivos y el embarazo actual, las cuales podían ser negativas, como también, experiencias positivas con sus hijos, siendo flexibles y tolerantes con ellos y disfrutándolos:

sin mucho apoyo de la pareja, difícil vivirlo sola, cambio de proyectos, volver a empezar, estresante. (Entrevistada 10)

\section{Relaciones interpersonales}

En este apartado se analizan los resultados con respecto a la relación que tenían las participantes con su pareja, su familia y sus redes de apoyo al momento del embarazo y a partir del diagnóstico de riesgo.

\subsection{Pareja}

La relación de pareja de las participantes en el estudio fue clasificada en dos subcategorías: estable (estaban casadas o vivían con la pareja desde hacía tiempo) o inestable (tenían una relación de poco tiempo ("seis meses o menos"), y no vivían juntos o no tenían pareja). Sin embargo, existieron diferencias en la percepción de la relación con respecto al tipo de riesgo médico de la gestación.

Ante el diagnóstico del embarazo de alto riesgo, la reacción de quienes tenían una relación estable fue de gusto e ilusión, pues percibían que sus parejas las apoyaban y las cuidarían para que el embarazo se lograra; quienes tenían conflictos con sus parejas o estaban separadas, percibieron una respuesta negativa de su parte, sobre todo de enojo, desconcierto y preocupación por la situación económica, e incluso se negaron a hacerse a responsables.

Al respecto, las mujeres con enfermedades crónicas previas refirieron que la relación con su pareja se vio afectada por las experiencias obstétricas negativas anteriores, por lo que en este embarazo ellos mostraban preocupación por la salud de ellas y por las posibles complicaciones que pudieran tener y al mismo tiempo, las cuidaban:

Pues él me apoya en todo y me procura para que yo esté bien, si yo me siento cansada, pues él hace las cosas, él cuida a la niña. (Entrevistada 1)

Las mujeres con relación de pareja inestable vivieron su embarazo solas porque ellos decidieron no hacerse responsables de su hijo.

Las parejas de estas mujeres reaccionaron de dos maneras:

i. con gusto, ilusión, apoyo y cuidado hacia su mujer frente al diagnóstico del embarazo, aunque al mismo tiempo con miedo de ponerlas en riesgo otra vez:

yo no te quiero poner en riesgo, o sea, ya pasaste por dos (pérdidas). (Entrevistada 4)

ii. negándose a hacerse responsables del embarazo inesperado:

Me dijo que si quería dinero, me daba dinero, pero que hasta ahí, que él no se quería hacer responsable. (Entrevistada 11)

Por su parte, las mujeres con complicaciones del embarazo que tenían una relación de pareja estable, percibían su relación distante y sin apoyo o, por el contrario, con apoyo y alegría por el nuevo embarazo; las mujeres con relación de pareja inestable percibían que su relación había cambiado a partir del embarazo:

sí yo siento que nos embarazamos muy rápido, nos embarazamos en seis meses de relación, pero no fue un accidente... (Entrevistada 5)

Pese a ello, el que algunas tomaran la decisión de embarazarse sin consultarlo con su pareja repercutió negativamente en su relación sentimental y en las reacciones que percibieron de ellos cuando se enteraron del embarazo.

Asimismo, las participantes con otros factores de riesgo con una relación de pareja estable señalaron que ésta se caracterizaba tanto por manifestaciones de enojo, reproches, falta de comprensión, pocas muestras de afecto y soledad, como por una buena relación, con apoyo y cuidados, aunque con algunos desacuerdos en la educación de los hijos. Quienes tenían una relación inestable de pareja vivieron modificaciones en su vida a partir del embarazo: se quedaron sin el apoyo de la pareja, quien no se hizo responsable del hijo, o decidieron vivir con su pareja a pesar de las dificultades económicas y los problemas de adicción que algunos tenían:

Pues no, que no, que no se iba a hacer cargo de los bebés tampoco. (Entrevistada 10)

En los tres grupos las reacciones de las parejas frente a las condiciones del embarazo fueron de apoyo o de rechazo a hacerse responsables, lo cual también provocaba mucho estrés a las mujeres.

\subsection{Familia}

Para las participantes de los tres grupos, la familia jugaba un papel importante de apoyo y sostén ante las experiencias obstétricas vividas previamente y las 
complicaciones presentadas en el embarazo actual, aunque también reconocían el impacto emocional que estas situaciones generaron en los miembros de su familia. Sin embargo, se vuelve a hacer evidente que el tipo de riesgo del embarazo también matiza la relación con la familia, quien se involucra y se siente afectada por las complicaciones tenidas y cuando el riesgo sólo es potencial, la familia no lo vivía con preocupación sino con gusto.

En este sentido, las entrevistadas con enfermedades crónicas previas percibían que sus familiares estaban preocupados por el riesgo y las complicaciones que presentaban en este embarazo. Les inquietaba la salud de la mujer y la del bebé y que ellos (papás, hermanos, hijos, etc.) tuvieran que hacerse cargo de los cuidados.

Porque les doy muchos sustos (a mis padres), dice (mi papá) "te pones muy mal y no", y mi mamá entonces esta vez que me enteré (del embarazo) no le quería decir a mi papá... (Entrevistada 1)

Algunas entrevistadas con complicaciones del embarazo percibían que con respecto al riesgo de su embarazo sus familiares se mostraban poco afectuosos y expresivos, distantes, o preocupados y aprensivos por su salud; no obstante, a ellas les inquietaba la salud de sus padres:

Mi mamá es muy aprensiva, está mal del corazón, cualquier noticia le trae problemas, lo que menos quiero es que ella se me ponga mal. (Entrevistada 5)

En cambio, las mujeres con otros factores de riesgo percibían a sus familiares contentos y que las apoyaban:

Todos bien contentos, mi suegra, mi mamá, todos mis cuñados, todos mis hermanos... (Entrevistada 6)

\subsection{Otras redes de apoyo}

Las participantes percibieron también que la gente que las rodea (vecinos y amigos) mostró cambios hacia ellas a partir de su embarazo.

Las mujeres con enfermedades previas mencionaron que algunas de sus amistades las apoyaron más durante su embarazo, sin embargo, hubo algunas que decidieron alejarse de ellas:

pues las amistades, eso sí, pues muchas me dejaron de hablar, quién sabe, no sé... (Entrevistada 11)

Por su parte las mujeres con complicaciones del embarazo expresaron que empezaron a sentirse más aceptadas por su comunidad a partir de la gestación:

se preocupan por mí, ya son muchos años de estar ahí y yo nunca me sentí integrada ahí a la comunidad. (Entrevistada 7)

Algunas participantes del grupo de otros factores de riesgo mencionaron haber percibido mayor interés de sus compañeros de trabajo a partir de su embarazo.

\section{Discusión}

La experiencia de malestar psicológico para las mujeres entrevistadas puede comprenderse a partir de dos momentos de crisis o epifanías: 1) el embarazo inesperado y 2 ) del tipo de riesgo médico diagnosticado.

Así, para las mujeres con enfermedades crónicas previas, el malestar se caracteriza por sentir miedo por su salud, ambivalencia entre la alegría y el temor, así como por la angustia y preocupación de volver a tener complicaciones y perder la gesta actual, ya que las malas experiencias de los embarazos previos les dejaron sensaciones de vacío y dolor. Algunas contaban con apoyo de su pareja, pero otras vivieron su maternidad solas. Contaban con el apoyo de su familia, aunque también les preocupaba afectarlos con este nuevo embarazo.

Por su parte, en algunas mujeres con complicaciones del embarazo el malestar psicológico se entiende por la angustia y el temor de volver a padecer una nueva pérdida, ya que habían tenido pérdidas gestacionales recurrentes. Esta situación llegó a afectar la relación con su pareja, con distanciamiento y poco apoyo, así como con dificultad para asumir la responsabilidad. Algunas contaban con el apoyo familiar, otras tenían que ocultar su complicación para no afectar la salud de sus padres.

En cuanto a las mujeres con otros factores de riesgo, éstas no vivieron su gestación como de alto riesgo, porque no existía ninguna enfermedad previa ni ninguna complicación, sino que su riesgo médico era sólo potencial. Aunque en este grupo la nueva gesta les provocó sorpresa y alegría, el malestar psicológico se hizo evidente a través del enojo y preocupación que sentían por su situación económica y para algunas, en la dificultad para aceptarlo. Los embarazos previos les generaron algunos problemas con la pareja y contaban con apoyo de la familia y amistades.

A lo largo de este trabajo, se ha visto que el tipo de riesgo médico presentado por estas mujeres es un aspecto que abre posibilidades adicionales de comprensión del fenómeno y lo que pudiera explicar las diferencias en la percepción de la experiencia y en la intensidad de las manifestaciones emocionales asociadas.

Al respecto, desde la percepción de estas mujeres, se obtuvieron sólo dos grupos de riesgo: aquellas que viven su embarazo sin riesgo (otros factores de riesgo: diagnosticadas médicamente con un riesgo potencial) y aquellas que lo viven como un embarazo de alto riesgo para su salud y la de su bebé (diagnosticadas médicamente como alto riesgo por enfermedades previas al embarazo actual y complicaciones del embarazo). Esto coincide con lo propuesto por Carolan (2008), quien afirma que para la mujer el riesgo se experimenta como un evento inusual equiparado con un potencial 
de pérdida o daño. Sin embargo, esto es contrario a lo que afirma Lupton (1999) acerca de que no existe el "no riesgo" del embarazo, ya que el potencial de daño siempre está presente.

Esta percepción del embarazo con o sin riesgo para las mujeres de este estudio depende de los siguientes aspectos: tener hijos vivos, no haber tenido pérdidas previas ni haber sido hospitalizadas, y contar con el apoyo de la pareja, la familia y personas cercanas. Esto concuerda con lo dicho por Carolan (2008), en cuanto a que, desde sus experiencias previas y del contexto en el que viven, estas mujeres no consideran que exista un riesgo en su embarazo. Estos aspectos también influyen para establecer una diferencia en la intensidad y la duración del malestar psicológico en la mujer con embarazo de alto riesgo.

Además, se pudo observar que frente a un riesgo inminente para la salud de la mujer y de su hijo, las manifestaciones emocionales más importantes fueron: miedo a la pérdida, enojo, tensión, incertidumbre, miedo a la soledad, angustia, miedo por la salud, miedo a volver a vivir la experiencia, devastación emocional, tal como lo plantean Thirlaway \& Heggs (2005), quienes afirman que las mujeres experimentan una reacción emotiva frente al riesgo. Sin embargo, también se observa que subyace un deseo de realizarse como mujer a través de la maternidad, tal y como lo afirma Saletti (2008), quien plantea que existe la creencia de que toda mujer no sólo es madre en potencia, sino que es madre en deseo y necesidad.

De acuerdo con lo analizado en este trabajo, para alcanzar una comprensión amplia del malestar psicológico debemos considerar, entre otros aspectos, la experiencia de la maternidad y las relaciones interpersonales que las mujeres viven durante la gestación.

Algunas mujeres con embarazo de alto riesgo, quienes han tenido pérdidas gestacionales previas, vivieron su maternidad como una experiencia dolorosa, con mucha angustia y miedo de perder el nuevo embarazo por complicaciones. Han aprendido que la maternidad es un proceso difícil y que implica sacrificios, incluso arriesgar su vida, y que además es el precio que tienen que pagar para ser madres y cumplir con su rol y con lo que se espera de ellas, y para preservar también su identidad, por lo que han integrado sus complicaciones a su vivencia de la maternidad, aunque también subyace en ellas el deseo de ser madres por encima de los riesgos que esto implique.

Lo anterior concuerda con lo planteado por Ramírez (1999) respecto a que a las mujeres se les educa desde pequeñas para aceptar el rol materno y se refuerza también en ellas la importancia de la relación madre-hijo; coincidiendo además con lo dicho por Saletti (2008) acerca de que la ideología patriarcal sitúa a las mujeres dentro del ámbito de la reproducción biológi- ca, negando su identidad fuera de la función materna.

Para algunas mujeres, las complicaciones vividas durante la gestación y el impacto emocional que les genera, pasan a ser parte de lo esperado y de lo cotidiano para ellas cada vez que se embarazan. Sin embargo, el hecho de que su pareja y su familia las acompañen y las apoyen durante el embarazo o decidan alejarse y no participar, contribuye de manera importante a que se exacerbe o disminuya la manifestación del malestar psicológico.

Por otra parte, llama la atención que las mujeres con un diagnóstico médico previo de alto riesgo no planearon sus embarazos, o más bien no los evitaron, a pesar del peligro que implicaba para su salud una nueva gestación (e incluso para su vida). Esta falta de planeación se ve reflejada en los relatos, en donde se aprecia que ellas parecen asumir una posición pasiva en la que dejan en manos de las circunstancias el volver a embarazarse o no ("por voluntad de Dios"), y no se cuidan, aunque su vida estuviese en riesgo. Aquí puede verse que subyace un deseo de ser madres y un pensamiento de "¿qué tal si ahora sí lo logro?", el cual es más fuerte que los riesgos que pudieran correr en una nueva gestación.

Esto hace pensar que, para ellas, "planear" un embarazo es una decisión voluntaria que va en contra de su función social e incluso de su religión, es decir, de lo que se espera de ellas. A nivel subjetivo, una explicación de ello podría encontrarse en lo propuesto por Burin (1990) con respecto a que la maternidad es la base de la identidad de las mujeres, y lo que "garantiza" su salud mental.

Desde esta perspectiva, es probable que estas mujeres se sigan embarazando porque persiste en ellas el deseo de ser madres, pero además no pueden ir en contra de su propia identidad ni de su rol como mujeres $y$, por tanto, de su salud mental, lo que les genera un conflicto subyacente. Este se da entre el mandato interiorizado de cumplir su función social como madres, que es lo que se espera de ellas socialmente y "así debe ser", y con ello preservar su identidad como mujeres; o cuidar su salud y evitar arriesgar su vida nuevamente, y en donde puede verse, implícitamente, que su identidad y su deseo son mucho más fuertes que su propia vida, tal y como lo afirma esta autora.

De igual modo, las relaciones interpersonales para estas mujeres tienen mucha importancia para la vivencia de su embarazo. Estas relaciones pueden llegar a ser incluso generadoras de malestar psicológico en las siguientes circunstancias: cuando por la secuela emocional de las experiencias previas negativas de otros embarazos, la relación de pareja se desgasta, se vuelve distante y no se puede hablar ni compartir el dolor vivido, llegando incluso a la separación o la infidelidad. Ante esto, la mujer tiene la creencia de que a través de 
un nuevo embarazo podrá retener a su pareja.

Otra circunstancia que influye en el malestar es cuando la pareja se niega a hacerse responsable del embarazo, enfrentando a la mujer a tener que vivir su maternidad sola. Por otro lado, también algunos hombres comparten la alegría y el temor del nuevo embarazo, en donde subyace un miedo de perder a su pareja si vuelven a presentarse complicaciones obstétricas.

Por su parte, la familia influye en la manifestación del malestar psicológico únicamente cuando la mujer no puede revelarles la gravedad de su condición en este embarazo, para no afectar su salud y evitarles preocupaciones (sobre todo a los padres que son mayores y están enfermos) y, por tanto, no puede recibir todo el apoyo que espera de su parte.

En cuanto a sus redes de apoyo, éstas tienden a mostrarles mayor apoyo y preocupación por su embarazo, aunque hay algunas personas que prefieren alejarse.

Para concluir, los hallazgos más importantes de este estudio fueron:

A. que en la medida en que las mujeres con embarazo de alto riesgo tienen más complicaciones obstétricas y pérdidas gestacionales, presentan mayor secuela emocional en la vivencia del embarazo actual y las manifestaciones emocionales son más intensas.

B. El malestar psicológico en las mujeres con embarazo de alto riesgo es un proceso que se da en un continuo, en el que todas pueden llegar a presentarlo en mayor o menor grado, a partir de la combinación de diferentes circunstancias que se desarrollan en el contexto de la vida cotidiana. De igual modo, quizás no podría hablarse de una condición "sin malestar", aunque las mujeres llegan a vivir momentos de ilusión, alegría y esperanza; no obstante, se observa que el malestar psicológico está presente durante el embarazo de alto riesgo, y su manifestación puede exacerbarse o atenuarse, a partir de los momentos de crisis, del contexto y del tipo de riesgo que se da en la gestación.

Finalmente, el explicar la experiencia emocional de las mujeres frente al embarazo de alto riesgo como un malestar psicológico, nos acerca más a lo propuesto por el enfoque biopsicosocial, desarrollado por Velasco (2006), quien plantea que los síndromes y síntomas físicos no sólo tienen su causa en la biología, sino también en la esfera simbólica, es decir, que proceden de la posición subjetiva de la persona en su contexto social y en la manera en que se enfrentan los conflictos.

Las autoras queremos agradecer y expresar nuestro reconocimiento a las mujeres entrevistadas que compartieron sus experiencias para hacer posible este trabajo.

\section{Referencias}

Austin, M. \& Priest, S. (2005). Clinical issues in perinatal mental health: new developments in the detection and treatment of perinatal mood and anxiety disorders. Acta Psychiatrica Scandinavica, 112(2), 97-104. doi:10.1111/j.16000447.2005.00549.x

Berenzon-Gorn, S., Saavedra, N. \& Alanís-Navarro, S. (2009). Estrategias utilizadas por un grupo de mujeres mexicanas para cuidar su salud emocional: autoatención y apoyo social. Salud Pública de México, 51(6), 474-481. Recuperado de http://ref.scielo.org/nyd3nh

Burin, M. (1990). El malestar de las mujeres, La tranquilidad recetada. Buenos Aires: Paidós.

Carolan, M. (2008). Toward understanding the concept of risk for pregnant women: some nursing and midwifery implications. Journal of Clinical Nursing, 18(5), 652-658. doi:10.1111/ j.1365-2702.2008.02480.x

Castro, R. (2000). La vida en la adversidad: el significado de la salud y la reproducción de la pobreza. Cuernavaca: UNAM / Centro Regional de Investigaciones Multidisciplinarias.

Creswell, J. (1998). Qualitative inquiry and research design. California: Sage.

Dennis, C-L. (2005). Psychosocial and psychological interventions for prevention of postnatal depression: systematic review. British Medical Journal, 331(15), 1-8. doi:10.1136/bmj.331.7507.15

Denzin, N. (2001). Interpretive interactionism. California: Sage Publications Inc.

Fisher, J., Feekery, C. \& Rowe-Murray, H. (2002). Nature, severity and correlates of psychological distress in women admitted to a private mother-baby unit. Journal of Paediatric Child Health, 38(2), 140-145. doi:10.1046/j.1440-1754.2002.00723.x

Flick, U. (2004). Introducción a la investigación cualitativa. Madrid: Morata.

Glazier, R., Elgar, F., Goel, V. \& Holzapfel, S. (2004). Stress, social support, and emotional distress in a community sample of pregnant women. Journal of Psychosomatic Obstetrics and Gynecology, 25, 247-255. doi:10.1080/01674820400024406

Gómez, M. (2007). Guía de intervención psicológica para pacientes con embarazo de alto riesgo. Perinatología y Reproducción Humana, 21(2), 111-121. Recuperado de http://www.medigraphic.com/pdfs/inper/ip-2007/ip072f.pdf

Gómez, M. \& Aldana, E. (2007). Alteraciones psicoló- 
gicas de la mujer con embarazo de alto riesgo. Psicología y Salud, 17(1), 53-61. Recuperado de http://revistas.uv.mx/index.php/psicysalud/article/view/739

Gómez, M., Morales, F., Aldana, E. \& Gaviño, F. (2008). Estado emocional de la mujer en relación con el parto o cesárea. Ginecología y Obstetricia de México, 76(7), 365-372. Recuperado de http:// www.imbiomed.com.mx/1/1/articulos.php?method=showDetail\&id articulo $=57873 \& i d$ seccion $=407 \&$ id ejemplar $=5858 \&$ id revista $=40$

Hediye, A. \& Korkmaz, N. (2005). The physical and psychological problems of hospitalized high-risk pregnant women y partial bed rest. Perinatal Journal, 13, 91-100. Recuperado de http://www. perinataljournal.com/20050132004

Kvale, S. (1996). InterViews. An introduction to qualitative research interviewing. California: SAGE Publications.

Lafaurie, M., Castañeda, K., Castro, D., Laverde, S., Balaguera, L., López, C., Martínez, E., Martínez, Y., Parra, C., Ramírez, N. \& Pardo, Y. (2011). Vivencia de gestantes con embarazo de alto riesgo. Revista Colombiana de Enfermería, 6(6), 15-28. Recuperado de http://www.uelbosque.edu. co/sites/default/files/publicaciones/revistas/ revista colombiana enfermeria/volumen6/vivencias gestantes.pdf

Lara, M., Navarro, C. \& Navarrete, L. (2010). Outcome results of a psycho-educational intervention in pregnancy to prevent PPD: A randomized control trial. Journal of Affective Disorders, 122(1-2), 109-117. doi:10.1016/j.jad.2009.06.024

Laza, C., Quintero, J., Jiménez, H. \& Preciado, J. (2013). Experiencias y sentimientos vividos durante una gestación de alto riesgo: un estudio documental 2005-2011. Enfermería Global, 12(3), 357371. doi:10.6018/eglobal.12.3.152821

Lupton, D. (1999). Risk and sociocultural theory. New directions and perspectives. Cambridge University Press.

Maraví, R. (2007). Contexto ético de la investigación social. Investigación educativa, 11(19), 137-151. Recuperado de http://revistasinvestigacion. unmsm.edu.pe/index.php/educa/article/ view/3624

Martínez, P. (2006). El método de estudio de caso. Estrategia metodológica de la investigación científica. Pensamiento y gestión, 20, 165-193. Recuperado de http://rcientificas.uninorte.edu.co/ index.php/pensamiento/article/view/3576

Meo, A. (2010). Consentimiento informado, anonimato y confidencialidad en investigación social. La experiencia internacional y el caso de la sociología en Argentina. Aposta Revista de Ciencias Sociales, 44, 1-30. Recuperado de http://www. apostadigital.com/revistav3/hemeroteca/aines.pdf

National Institute of Health. (2008). Protección de los participantes humanos de la investigación. (En red.). Recuperado de http://pphi.nihtraining. $\mathrm{com} /$

Peixoto, M., Carvalho, C. \& Lefévre, F. (2006). Os significados da maternidade para mulheres cardiopatas e diabéticas com gravidez de risco. Revista brasileira de crescimento e desenvolvimento humano, 16(1), 12-21. doi:10.7322/jhgd.19777

Pinho, A., Pamplona, V. \& Garcia de Lima, C. (2010). Sentimientos y percepciones de mujeres en el ciclo embarazo-puerperio que sobrevivieron a una morbosidad materna grave. Revista Latino-Americana Enfermagem, 18(6), 1-8. Recuperado de http://www.scielo.br/pdf/rlae/v18n6/ es 20.pdf

Ramírez, S. (1999). El mexicano, psicología de sus motivaciones. México, D.F.: Grijalbo.

Saletti, L. (2008). Propuestas teóricas feministas en relación al concepto de maternidad. Clepsydra, 7, 169-183. Recuperado de http://publica.webs.ull.es/upload/REV\%20CLEPSYDRA/07-2008/11\%20Saletti.pdf

Stake, R. (2005). Investigación con estudio de casos. Madrid: Morata.

Thirlaway, K. \& Heggs, D. (2005). Interpreting risk messages: Women's response to health story. Health, Risk \& Society, 7(2), 107-121. doi:10.1080/13698570500108677

Velasco, S. (2006). Evolución de los enfoques de género en salud y teoría feminista. Madrid, España: Escuela Nacional de Sanidad y Observatorio de Salud de la Mujer del $\mathrm{M}^{\circ}$ de Sanidad y Consumo e Instituto de la Mujer del $\mathrm{M}^{\circ}$ de Trabajo y Asuntos Sociales. 